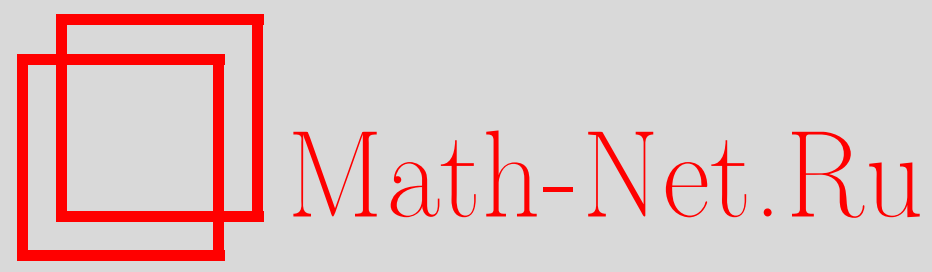

Г. Г. Амосов, В. Ж. Сакбаев, О самосопряженных расширениях оператора Шрёдингера с вырождением на двух полупрямых и определяемых ими марковских коциклах, $M a-$ тем. заметки, 2004, том 76, выпуск 3, 335-343

DOI: https://doi.org/10.4213/mzm107

Использование Общероссийского математического портала Math-Net.Ru подразумевает, что вы прочитали и согласны с пользовательским соглашением http://www.mathnet.ru/rus/agreement

Параметры загрузки:

IP : 54.209 .52 .79

26 апреля 2023 г., $12: 37: 50$

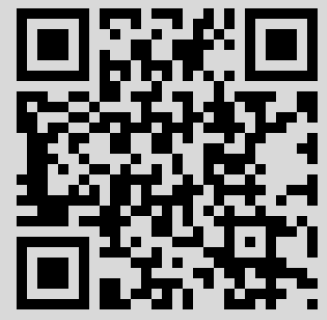




\title{
О САМОСОПРЯЖЕННЫХ РАСШИРЕНИЯХ ОПЕРАТОРА ШРЁДИНГЕРА С ВЫРОЖДЕНИЕМ НА ДВУХ ПОЛУПРЯМЫХ И ОПРЕДЕЛЯЕМЫХ ИМИ МАРКОВСКИХ КОЦИКЛАХ
}

\author{
Г. Г. Амосов, В. Ж. Сакбаев
}

Рассматривается уравнение Шрёдингера на прямой для частицы с массой, зависящей от положения частицы. Исследуется корректная постановка задачи Коши для уравнения Шрёдингера с вырожденным оператором, характеристическая форма которого обращается в нуль вне некоторого отрезка $I=[-l, l]$ на прямой $\mathbb{R}$. Показано, что такая задача порождает унитарный марковский коцикл.

Библиография: 19 названий.

Введение. В настоящей работе изучаются задачи, связанные с описанием динамики квантовых механических систем, эффективная масса которых зависит от положения в координатном пространстве. Примеры таких систем возникают в физике твердого тела при описании движения электрона в неоднородной среде (металл - полупроводник) (см. [1, гл. 9]), и при описании движения систем со связями (см. [2]). В случае, когда эффективная масса системы обрашается в нуль в некоторых точках координатного (или фазового) пространства, описание динамики механической системы уравнениями классической механики становится некорректным (см. [2]). Мы осуществляем попытку описать движение таких систем с помощью уравнения Шрёдингера, гамильтониан которого не является равномерно эллиптическим оператором, а вырождается в некоторых точках координатного пространства. Мы устанавливаем, что решение такого уравнения определяет унитарный марковский коцикл в смысле определения [3]. То есть, вторичное квантование этого коцикла в бозонном пространстве Фока является марковским коциклом в смысле согласованности с естественной фильтрацией фоковского пространства (см. [4], [5] и содержащиеся там ссылки). Таким образом, из рассматриваемого примера видно, что для уравнений Шрёдингера с вырождением должно быть эффективно квантовое стохастическое исчисление.

В работе изучается корректность постановки задачи Коши для уравнения Шрёдингера с гамильтонианом, являющимся дифференциальным оператором переменного типа,

$$
i \frac{\partial u(t, x)}{\partial t}=\mathbf{L} u(t, x), \quad t>0, \quad x \in \mathbb{R} .
$$


Уравнение (1) дополнено начальньм условием

$$
u(+0, x)=u_{0}(x)
$$

ниже будет указано, в каком смысле выполнено условие (2).

Пусть координатное пространство $\mathbb{R}$ разделено на три области $\left.\omega_{1}: x<-l, \omega_{2}: x\right\rangle l$ и $\Omega: x \in[-l, l]$ границами $\gamma_{1}: x=-l$ и $\gamma_{2}: x=l, l>0$. Исследуем задачу (1), (2) с оператором $\mathbf{L}$, вырождающимся в областях $\omega_{1}$ и $\omega_{2}$. Действие оператора $\mathbf{L}$ задается формулой

$$
\mathbf{L} v=-\frac{\partial}{\partial x}\left[\epsilon(x) \frac{\partial v}{\partial x}\right]+\frac{i}{2}\left(a(x) \frac{\partial v}{\partial x}+\frac{\partial a(x) v}{\partial x}\right),
$$

где $\epsilon(x)=\chi_{l}(x), a(x)=a\left(1-\chi_{l}(x)\right), a \in \mathbb{R} ;$ здесь $\chi_{l}(x)$ - характеристическая функция отрезка $[-l, l]$.

Заметим, что изучение вопросов о корректности постановки начально-краевых задач для эллиптико-параболических уравнений второго порядка проводилось в работе [6], где были указаны области задания краевых условий в зависимости от вида дифференциального оператора. Исследование задачи Коши типа (1), (2) для уравнения Шрёдингера с оператором переменного типа, вырождающимся на полупрямой, проводилось в работах [7], [8], в которых была сформулирована постановка задачи Коши и получены необходимые и достаточные условия сушествования и единственности ее решения.

1. Постановка задачи. Зададим максимальную область определения оператора $\mathbf{L}$, состоящую из таких функций $u$, что $\mathbf{L} u \in L_{2}(\mathbb{R})$. Область определения $D(\mathbf{L})$ оператора $\mathbf{L}$ включается в линейное пространство $M$ функций, сужение которых на множество $\omega_{l}=(-\infty,-l) \cup(l,+\infty)$ принадлежит пространству $W_{2}^{1}(\omega)$, а сужение на $\Omega_{l}=[-l, l]$ принадлежит пространству $W_{2}^{2}\left(\Omega_{l}\right)$ (см. $[9$, гл. 3]). Тогда функции из области определения оператора $\mathbf{L}$ и их первые производные имеют (см. [9, гл. 3]) следы $u(-l \pm 0), u(l \pm 0), u^{\prime}(l-0), u^{\prime}(l-0)$ на гранище $\gamma_{+}=\{-l, l\}$ областей $\Omega_{l}$ и $\omega_{l}$.

Укажем такое линейное многообразие $M^{\prime} \subset M$, что для любого $u \in M^{\prime}$ вьполняется включение $\mathbf{L} u \in D^{\prime}(\mathbb{R})$, где $D^{\prime}(\mathbb{R})$ есть пространство обобщенных функций на оси $\mathbb{R}$ (см. $[10$, гл. 2]). Для того, чтобы произведение функции $\epsilon(x)$ с разрывом на границе $\gamma$ и функции $d u(x) / d x$ являлось элементом пространства $D^{\prime}(\mathbb{R})$, необходимо и достаточно выполнение следующих условий:

$$
M^{\prime}=\{u \in M: u(-l-0)=u(-l+0), u(l-0)=u(l+0)\} .
$$

Рассмотрим оператор $\mathbf{L}$ как линейньй оператор из линейного пространства $M^{\prime}$ в линейное топологическое пространство $D^{\prime}(\mathbb{R})$. Для того, чтобы действие оператора $\mathbf{L}$ на функцию $u \in M^{\prime}$ являлось элементом $\mathbf{L} u$ пространства $L_{2}(\mathbb{R})$, выделим такое линейное подпространство $D(\mathbf{L}) \subset M^{\prime}$, что для любого $u \in D(\mathbf{L})$ обобщенная функция $\mathbf{L} u=g \in D^{\prime}(\mathbb{R})$ регулярна и имеет плотность $g(x) \in L_{2}(\mathbb{R})($ см. [10, гл. 2]). С помощью интегрирования по частям нетрудно получить, что для этого необходимо и достаточно выполнение условия

$$
D(\mathbf{L})=\left\{u \in M^{\prime}: u_{x}^{\prime}(-l+0)=i \frac{a}{2} u(-l-0), u_{x}^{\prime}(l-0)=i \frac{a}{2} u(l+0)\right\} .
$$


Тогда оператор $\mathbf{L}$ плотно определен на $L_{2}(\mathbb{R})$. C помощью интегрирования по частям нетрудно проверить, что условие (5) при выполнении условия (4) обеспечивает симметричность оператора $\mathbf{L}$ на пространстве $L_{2}(\mathbb{R})$.

Заметим, что оператор $\mathbf{L}$ на линейном многообразии $D(\mathbf{L})$ не является положительным, ибо $(u, \mathbf{L} u)=0$ для всех гладких вещественнозначных функций с носителем на положительном множестве $\Omega_{l}$. Кроме того, оператор $\mathbf{L}$ не является самосопряженным, но обладает самосопряженньми расширениями, так как имеет одинаковые индексы дефекта, равные единище.

Найдем область определения сопряженного оператора $\mathbf{L}^{*}$. С помощью интегрирования по частям выражения $\left(\mathbf{L}^{*} v, u\right), v \in D\left(\mathbf{L}^{*}\right), u \in \mathbf{L}$, равного выражению $(v, \mathbf{L} u)$, для таких $u \in D(\mathbf{L})$, что $\operatorname{supp}(u) \subset \omega_{l}$ или $\operatorname{supp}(u) \subset \Omega_{l}$, заключаем, что $D\left(\mathbf{L}^{*}\right) \subset M$ (см. также $[11$, гл. 8, п. 2]). Тогда функции $u(x) \in D(\mathbf{L})$ имеют предельные значения $u(l \pm 0), u_{x}^{\prime}(l-0), u(-l \pm 0), u_{x}^{\prime}(-l+0)$ и нетрудно проверить, что область определения оператора $\mathbf{L}^{*}$ есть

$$
\begin{gathered}
D\left(\mathbf{L}^{*}\right)=\left\{u:\left.u\right|_{\Omega_{l}} \in W_{2}^{2}\left(\Omega_{l}\right),\left.u\right|_{\omega_{l}} \in W_{2}^{1}\left(\omega_{l}\right),-i u_{x}^{\prime}(l-0)+i \frac{a}{2} u(l-0)=i a u(l+0)\right. \\
\left.u_{x}^{\prime}(-l+0)+i \frac{a}{2} u(-l+0)=i a u(-l-0)\right\}
\end{gathered}
$$

Оператор $\mathbf{L}^{*}$ каждой функции $u(x)$ из $D\left(\mathbf{L}^{*}\right)$ сопоставляет функцию $\mathbf{L}^{*} u(x) \in L_{2}(\mathbb{R})$, задаваемую условиями

$$
\left.\mathbf{L}^{*} u(x)\right|_{\omega_{l}}=\left.i a \frac{\partial}{\partial x} u(x)\right|_{\omega_{l}},\left.\quad \mathbf{L}^{*} u(x)\right|_{\Omega_{l}}=-\left.\frac{\partial^{2}}{\partial x^{2}} u(x)\right|_{\Omega_{l}} .
$$

Тогда можно непосредственно проверить, что если $a \neq 0$, то $\operatorname{Ker}\left(\mathbf{L}^{*}-i a \mathbf{I}\right)$ и $\operatorname{Ker}\left(\mathbf{L}^{*}+\right.$ iaI) есть нетривиальные одномерные линейные подпространства, а именно:

$$
\operatorname{Ker}\left(\mathbf{L}^{*}+i \mathbf{I}\right)=\operatorname{Lin}\left(\varphi_{+}(x)\right), \quad \operatorname{Ker}\left(\mathbf{L}^{*}-i \mathbf{I}\right)=\operatorname{Lin}\left(\varphi_{-}(x)\right),
$$

где $\left.\varphi_{+}(x)\right|_{(-\infty,-l)}=0,\left.\varphi_{+}(x)\right|_{(l, \infty)}=e^{-x}$ и $\left.\varphi_{+}(x)\right|_{\Omega_{l}}$ есть решение неоднородной третьей краевой задачи на отрезке $\Omega_{l}$ :

$$
\begin{gathered}
-\frac{\partial^{2} \varphi_{+}}{\partial x^{2}}+i \varphi_{+}=0, \quad x \in \Omega_{l}, \\
\varphi_{+}^{\prime}(-l+0)+i \frac{a}{2} u(-l+0)=0, \quad \varphi_{+}^{\prime}(l-0)+i \frac{a}{2} u(l-0)=1,
\end{gathered}
$$

которая, как несложно проверить, имеет единственное решение. Ядро $\operatorname{Ker}\left(\mathbf{L}^{*}-i \mathbf{I}\right)$ устроено аналогично.

Определим, какие самосопряженные расширения допускает симметрический оператор $\mathbf{L}$ на $D(\mathbf{L})$. Область определения оператора $\mathbf{L}$ задана как подпространство в пространстве $M$ с помощью четырех линейных связей на шесть граничных значений функции и ее производной на гранищах раздела типа оператора, а область определения опеpaтора $\mathbf{L}^{*}$ задана как подпространство в пространстве $M$ с помощю двух таких линейных связей. Поэтому самосопряженное расширение оператора $\mathbf{L}$ следует задать как 
подпространство в пространстве $D\left(\mathbf{L}^{*}\right)$ с помощью одного уравнения линейной связи на четыре независимых граничных значения $u(l \pm 0), u(-l \pm 0)$ функции и ее производной на границах раздела типа оператора $\mathbf{L}^{*}$ (так как две связи

$$
u^{\prime}( \pm(l-0))=i a\left(u( \pm(l+0))-\frac{1}{2} u( \pm(l-0))\right)
$$

уже наложены на функции из $\left.D\left(\mathbf{L}^{*}\right)\right)$.

Будем искать самосопряженное расширение $\mathscr{L}$ оператора $\mathbf{L}$ в виде сужения оператора $\mathbf{L}^{*}$ на следующее линейное многообразие:

$$
D(\mathscr{L})=\left\{u \in D\left(\mathbf{L}^{*}\right): u(l+0)=\alpha_{1} u(l-0)+\alpha_{2} u(-l+0)+\alpha_{3} u(-l-0)\right\}
$$

где $\alpha_{j}, j \in \overline{(1,3)}$ - неопределенные постоянные. Тогда ограничения на коэффициенты $\alpha_{i}, i=1,2,3$, обеспечивающие самосопряженность $(\mathscr{L} u, v)=(u, \mathscr{L} v), u, v \in D(\mathscr{L})$ принимают вид $\alpha_{1}=1, \alpha_{2}=-\alpha_{3},\left|\alpha_{3}\right|=1$, т.е.

$$
u(l+0)-u(l-0)=e^{i \varphi}(u(-l+0)-u(-l-0)), \quad \varphi \in[0,2 \pi] .
$$

Положим $\mathscr{L}=\mathscr{L}_{\varphi}, D\left(\mathscr{L}_{\varphi}\right)=\left\{u \in D\left(\mathbf{L}^{*}\right): u(l+0)-u(l-0)=e^{i \varphi}(u(-l+0)-u(-l-0))\right\}$. Оператор $\mathscr{L}_{\varphi}$ каждой функции $u(x)$ из $D(\mathscr{L})$ сопоставляет функцию $\mathscr{L}_{\varphi} u(x) \in L_{2}(\mathbb{R})$, задаваемую условием $\mathscr{L}_{\varphi} u(x)=\mathbf{L}^{*} u(x)$.

Тогда при каждом $\varphi \in[0,2 \pi]$ получаем $D(\mathbf{L}) \subset D\left(\mathscr{L}_{\varphi}\right) \subset D\left(\mathbf{L}^{*}\right), \varphi \in[0,2 \pi]$ и оператор $\mathscr{L}_{\varphi}$ является самосопряженным расширением оператора $\mathbf{L}$.

Фиксируем некоторое $\varphi \in[0,2 \pi]$ и превратим область определения $D\left(\mathscr{L}_{\varphi}\right)$ в гильбертово пространство, снабдив его нормой графика оператора $\mathscr{L}_{\varphi}$.

ОПРЕДЕЛЕНИЕ 1. Решением задачи (1), (2) назовем функцию

$$
u(t, x) \in C\left(\mathbb{R}_{+}, D\left(\mathscr{L}_{\varphi}\right)\right) \cap C^{1}\left(\mathbb{R}_{+}, L_{2}(\mathbb{R})\right),
$$

которая удовлетворяет удовлетворяет уравнению (1) почти всюду на $\mathbb{R}_{+} \times \mathbb{R}$ и условию $(2)$ в том смысле, что $\left\|u(t, x)-u_{0}(x)\right\|_{L_{2}(\mathbb{R})} \rightarrow 0$ при $t \rightarrow+0$.

ОПРЕДЕЛЕНИЕ 2. Функцию $u(t, x) \in C\left(\mathbb{R}_{+}, L_{2}(\mathbb{R})\right)$ назовем обобщенным решением задачи (1), (2), если существует последовательность начальных условий $\left\{u_{0, n}(x)\right\}$,

$$
\lim _{n \rightarrow \infty}\left\|u_{0, n}(x)-u_{0}(x)\right\|_{L_{2}(\mathbb{R})}=0
$$

такая, что при каждом $n \in \mathbb{N}$ существует решение $u_{n}(t, x)$ задачи $(1),(2)$ с начальньми данньми $u_{0, n}(x)$ и выполняется условие

$$
\lim _{n \rightarrow \infty}\left\|u_{n}(t, x)-u(t, x)\right\|_{C\left(\mathbb{R}_{+}, L_{2}(\mathbb{R})\right)}=0 .
$$

Заметим, что если $u(t, x)$ есть обобщенное решение задачи $(1),(2)$, то

$$
\lim _{t \rightarrow+0}\left\|u(t, x)-u_{0}(x)\right\|_{L_{2}(\mathbb{R})}=0 .
$$


Действительно,

$$
u(t, x)-u_{0}(x)=u(t, x)-u_{n}(t, x)+u_{n}(t, x)-u_{0, n}(x)+u_{0, n}(x)-u_{0}(x),
$$

откуда следует требуемое утверждение.

Кроме того, нетрудно показать, что если $u(t, x)$ есть решение задачи $(1),(2)$, то оно удовлетворяет интегральному тождеству

$$
-i \int_{0}^{T}\left(\mathbf{L}^{*} v, u(t)\right) d t=(v, u(T))-(v, u(0)) \quad \forall T>0
$$

при любом выборе $v(x) \in D\left(\mathbf{L}^{*}\right)$.

2. Основные результаты о корректной разрешимости вырожденной задачи $(1),(2)$. Напомним, что мы выбрали некоторое значение $\varphi \in[0,2 \pi]$ и, тем самым, некоторое самосопряженное расширение $\mathscr{L}_{\varphi}$ оператора $\mathbf{L}$.

Поскольку оператор $\mathscr{L}_{\varphi}$ самосопряжен, из теоремы Стоуна (см. [1]) вытекает теоремa 1.

Tеорема 1. Пусть выполнено условие $u_{0} \in L_{2}(\mathbb{R})$. Тогда, если а $\in \mathbb{R}$, то задача (1), (2) имеет единственное обобщенное решение $u(x, t)$, причем $\|u(t, x)\|_{L_{2}(\mathbb{R})}=$ $\left\|u_{0}\right\|_{L_{2}(\mathbb{R})}$.

ЗАмечАниЕ. Обобщенное решение задачи $(1),(2)$ определяется с помощью грушы унитарных преобразований $\widetilde{U}=\left(\widetilde{U}_{t}\right)_{t \in \mathbb{R}}$ пространства $L_{2}(\mathbb{R})($ см. [10]):

$$
u(t, x)=\widetilde{U}_{t} u_{0}(x), \quad \widetilde{U}_{t}=\exp \left(i \mathscr{L}_{\varphi} t\right), \quad t \in \mathbb{R} .
$$

Из теоремы о спектральном разложении самосопряженного оператора $\mathscr{L}_{\varphi}$ вытекает следствие 1.

СлеДСтвиЕ 1. Пусть $a \in \mathbb{R}, m \in \mathbb{N}$ и функиия $u_{0}(x)$ такова, что $u_{0} \in D\left(\mathscr{L}_{\varphi}^{m}\right)$. Тогда существует единственное решение $u(t, x)$ задачи (1), (2), которое принадлежит пространству $C\left(\mathbb{R}_{+}, D\left(\mathscr{L}_{\varphi}^{m}\right)\right) \cap C^{m}\left(\mathbb{R}_{+}, L_{2}(\mathbb{R})\right)$ и причем

$$
\begin{gathered}
\|u(x, t)\|_{L_{2}(\mathbb{R})}=\left\|u_{0}(x)\right\|_{L_{2}(\mathbb{R})}, \\
\|u(x, t)\|_{C\left(\mathbb{R}_{+}, D\left(\mathscr{L}_{\varphi}^{m}\right)\right) \cap C^{m}\left(\mathbb{R}_{+}, L_{2}(\mathbb{R})\right)}=\left\|u_{0}(x)\right\|_{D\left(\mathscr{L}_{\varphi}^{m}\right)} .
\end{gathered}
$$

Здесь и далее через $C^{m}(\Delta, X)$ и $D\left(\mathscr{L}_{\varphi}^{m}\right)$ обозначается пространство $m$ раз непрерьвно дифференцируемых отображений промежутка $\Delta$ в линейное нормированное пространство $X$ и область определения $m$-й степени оператора $\mathscr{L}_{\varphi}$ соответственно. 
ДоКАЗАТЕЛЬСТво СЛЕДСТВИЯ. Единственность обобщенного решения следует из теоремы 1. Сушествование и гладкость решения, выражаемая соотношением (12), вытекают из утверждений теоремы 1 для задачи $(1),(2)$ с начальньми условиями $\mathscr{L}_{\varphi}^{k} u_{0}(x)$, $k=0, \ldots, m$. Следствие доказано.

Пусть $u_{0}(x) \in D\left(\mathscr{L}_{\varphi}^{m}\right)$ и $a<0$. Заметим, что тогда из $(12)$ при $m=2$ следует непрерьвная дифференцируемость решения $u(t, x)$ в областях $D_{-}=\mathbb{R} \times(-\infty,-l)$ и $D_{+}=\mathbb{R} \times(l,+\infty)$, и что сужение решения на область $D_{-}$удовлетворяет соотношениям

$$
\begin{array}{cl}
\left\|u(t, x)-u_{0}(x+a t)\right\|_{C\left(\mathbb{R}_{+}, L_{2}(-\infty,-l)\right)}=0, & t \geqslant 0, \\
\left\|u(t, x)-u_{0}(x+a t)\right\|_{C\left(\mathbb{R}_{-}, L_{2}(l,+\infty)\right)}=0, & t<0 .
\end{array}
$$

Поэтому в силу плотности линейного многообразия $D\left(\mathscr{L}_{\varphi}\right)$ в пространстве $L_{2}(\mathbb{R})$ и соотношения (11) равенства (13), (14) справедливы для обобщенного решения задачи (1), $(2)$ с любым начальньм условием $u_{0}(x) \in L_{2}(\mathbb{R})$.

Аналогично, если $a>0$, то имеют место равенства

$$
\begin{aligned}
\left\|u(t, x)-u_{0}(x+a t)\right\|_{C\left(\mathbb{R}_{+}, L_{2}(l, \infty)\right)}=0, & t \geqslant 0, \\
\left\|u(t, x)-u_{0}(x+a t)\right\|_{C\left(\mathbb{R}_{-}, L_{2}(-\infty,-l)\right)} & =0, \quad t<0 .
\end{aligned}
$$

ЗАмЕчАниЕ. В работах [7], [8] проводилось изучение корректной разрешимости задачи типа (1), (2) с оператором переменного типа, характеристическая форма которого вырождается на полуоси прямой $\mathbb{R}$. Тогда, как показано в работе [7], оператор $\mathbf{L}$ не имеет самосопряженных расширений. Это обстоятельство послужило причиной дать в работе [7] определение решения, отличающееся от определения 1 настоящей работы (в некотором смысле, более ограничительное: в определении 1 настоящей работы требуется принадлежность решения области определения расширения оператора $\mathbf{L}$, a в определении 1 работы [7] - области определения самого оператора L). Поэтому в зависимости от коэффициента при "младшем" члене оператора $\mathbf{L}$ задача Коши типа (1), (2) в работе [7] имеет решение не при любом начальном условии. В работе [8] проводится исследование корректности задачи $(1),(2)$ при различных обобщениях определения ее решения. А в настоящей работе для любого самосопряженного расширения $\mathscr{L}$ оператора $\mathbf{L}$ теорема 1 утверждает существование и единственность решения задачи $(1),(2)$ при любом начальном условии из пространства $L_{2}(\mathbb{R})$, однако выбор самосопряженного расширения и, тем самым, группы 'U унитарных преобразований пространства $L_{2}(\mathbb{R})$, не является однозначным.

3. Унитарные марковские коциклы порождаемые вырожденной задачей $(1),(2)$. Пусть $U=\left(U_{t}\right)_{t \in \mathbb{R}}$ есть сильно непрерьвная группа унитарных операторов в гильбертовом пространстве $\mathscr{H}$. Сильно непрерывное семейство $W=\left(W_{t}\right)_{t \in \mathbb{R}}$ ограниченных операторов в $\mathscr{H}$ назьвается (мультипликативным) $U$-коциклом, если вьполнено условие $W_{t+s}=W_{t} U_{t} W_{s} U_{-t}, s, t \in \mathbb{R}$. Коцикл $W$ назьвается унитарным, если все операторы $W_{t}$ унитарные. Теория коциклов широко используется в квантовой теории вероятностей, где в качестве $\mathscr{H}$ обычно берется симметричное (бозонное) Фоковское пространство $\Gamma(\mathscr{K})$ над одночастичным гильбертовым пространством $\mathscr{K}=L_{2}(\mathbb{R})$, 
а в качестве $U$ - унитарная группа в $\mathscr{H}$, полученная вторичньп квантованием группы сдвигов в $\mathscr{K}$ (см. [12]). Обыкновенно рассматриваются квантовые стохастические дифференциальные уравнения, решениями которых являются коциклы (см. [12]-[14]). Отметим, что квантовое стохастическое дифференциальное уравнение унитарно эквивалентно краевой задаче в фоковском комплексе (см. [13]). Часто выделяется подкласс марковских коциклов, удовлетворяющих специальному условию локализации действия (см. [5]-[6] и приведенные там ссылки). Другим направлением исследования является изучение возможности дилатации коциклов, состояших из сжимающих операторов в гильбертовом пространстве $\mathscr{H}$ до унитарных коциклов в некотором гильбертовом пространстве $\mathscr{H}^{\prime}, \mathscr{H} \subset \mathscr{H}^{\prime}$ (см. [15]). К унитарным коциклам можно подойти с точки зрения теории возмущений групп унитарных операторов следующим образом. Пусть $U=\left(U_{t}\right)_{t \in \mathbb{R}}$ и $\widetilde{U}=\left(\widetilde{U}_{t}\right)_{t \in \mathbb{R}}$ есть две сильно непрерьвных группы унитарных операторов в $\mathscr{H}$. Тогда семейство операторов $W_{t}=\widetilde{U}_{t} U_{-t}, t \in \mathbb{R}$, является $U$-коциклом. Группу $\widetilde{U}$ естественно в этом случае назвать коциклическим возмущением группы $U$ коциклом $W$. Поскольку любые две группы унитарных операторов связаны некоторым унитарным коциклом, для того, чтобы задача о коциклическом возмущении фиксированной группы была содержательной, нужно ограничить класс используемых при этом возмущении коциклов. Ниже мы рассмотрим задачу о коциклическом возмущении группы сдвигов в $\mathscr{K}$ коциклами, являющимися марковскими в смысле определения [4]. Коциклы в $\mathscr{K}$, марковские в смысле [4] (см. определение ниже), порождают марковские коциклы в $\mathscr{H}=\Gamma(\mathscr{K})$ в ходе процедуры вторичного квантования (см. [16]). Ниже мы будем систематически использовать основные факты теории инвариантных подпространств сдвигов (см. [17]).

Рассмотрим группу сдвигов $U$, действующую в пространстве $\mathscr{K}$ по формуле

$$
\left(U_{t} f\right)(x)=f(x+t), \quad f \in \mathscr{K}, \quad t, x \in \mathbb{R} .
$$

Пусть подпространство $\mathscr{K}(t) \subset \mathscr{K}$ состоит из функций с носителем, принадлежащим интервалу $[-t,+\infty)$. При процедуре вторичного квантования семейство подпространств $\mathscr{K}(t), t \geqslant 0$, задает естественную фильтрацию $\mathscr{H}_{t]}=\Gamma(\mathscr{K}(t)), \mathscr{H}_{[t}=\Gamma\left(\mathscr{K}(t)^{\perp}\right), t \geqslant 0$, фоковского пространства $\mathscr{H}=\Gamma(\mathscr{K})$ такую, что $\mathscr{H} \approx \mathscr{H}_{t]} \otimes \mathscr{H}_{[t}$. Унитарньй $U$-коцикл $W$ назьвается марковским, если $\left.W_{t}\right|_{\mathscr{K}(t) \perp}=I, t \geqslant 0$ (см. [4], [16]). Условие коцикла приводит к вьполнению тождества $W_{-t}=U_{-t} W_{t}^{*} U_{t}, t \geqslant 0$. Следовательно, для того чтобы коцикл $W$ был марковским необходимо и достаточно, чтобы вьполнялось тождество (см. [4])

$$
\left.W_{-t}\right|_{\mathscr{K}(0)^{\perp}}=I, \quad t \geqslant 0 .
$$

Вторично квантованный марковский коцикл $\Gamma\left(W_{t}\right)$ является марковским в смысле согласованности с фильтрацией $\mathscr{H}_{t]}, t \geqslant 0$, так что $\Gamma\left(W_{t}\right) \approx \mathscr{W}_{t} \otimes I, t \geqslant 0$, по отношению к представлению $\mathscr{H} \approx \mathscr{H}_{t]} \otimes \mathscr{H}_{[t}$.

Пространство $\mathscr{K}$ естественньм образом разбивается в ортогональную сумму пространств $\mathscr{K}_{+}$и $\mathscr{K}_{-}$, состоящих из функций с носителем, принадлежащим $\mathbb{R}_{+}$и $\mathbb{R}_{-}$соответственно. Аналитическая функция $\Theta(\lambda)$, голоморфная в полуплоскости $\operatorname{Im} \lambda>0$, назьвается внутренней, если $|\Theta(x)|=1, x \in \mathbb{R}$. Обозначим $M_{\Theta}=\mathscr{F}^{-1} \Theta \mathscr{F}-$ изометрический оператор в пространстве $\mathscr{K}_{+}$· Здесь $\mathscr{F}$ - преобразование Фурье, переводящее 
пространство $\mathscr{K}_{+}$в пространство Харди $H^{2}$ в полуплоскости $\operatorname{Im} \lambda>0$, где оператор умножения на функцию $\Theta(\lambda)$ является изометрическим. Оператор $M_{\Theta}$ обладает свойством $U_{-t} M_{\Theta} f=M_{\Theta} U_{-t} f, f \in \mathscr{K}_{+}, t \geqslant 0$, и определяет подпространство $\mathscr{K}_{\Theta}=M_{\Theta} \mathscr{K}_{+}$, инвариантное относительно сдвигов вправо $U_{-t}, t \geqslant 0$. Обозначим $V^{+}=\left(V_{t}^{+}\right)_{t \geqslant 0}$ и $V^{-}=\left(V_{t}^{-}\right)_{t \geqslant 0}$ полугруппы изометрических операторов в пространствах $\mathscr{K}_{\Theta}$ и $\mathscr{K}_{-}$, где

$$
V_{t}^{+}=U_{-t}\left|\mathscr{K}_{\Theta}, \quad V_{t}^{-}=U_{t}\right|_{\mathscr{K}_{-}}, \quad t \geqslant 0
$$

Поскольку полугруппы $V^{+}{ }_{\text {и }} V^{-}$состоят из сдвигов, т.е. $\wedge_{n \in \mathbb{N}} V_{t n}^{+} \mathscr{K}_{\Theta}=\wedge_{n \in \mathbb{N}} V_{t n}^{-} \mathscr{K}_{-}=$ $\{0\}$ для любого $t>0$, и вьполнено $(\mathrm{C})$, групша сдвигов $U$ называется унитарныл сиеллением полугрупп $V^{+}$и $V^{-}$в пространстве $\mathscr{K}$ (см. [18], [19]). Другим сцеплением $V^{+}$ и $V^{-}$является група $\widetilde{U}=\left(\widetilde{U}_{t}\right)_{t \in \mathbb{R}}$ в пространстве $\widetilde{\mathscr{K}}=\mathscr{K}_{\Theta} \oplus \mathscr{K}_{-}$, действующая следующим образом:

$$
\begin{aligned}
& \widetilde{U}_{t} f=U_{t} f, \quad f \in \mathscr{K}_{-}, \quad t \geqslant 0, \\
& \widetilde{U}_{t} f=U_{t} P_{[0, t]} g+M_{\Theta} U_{t} P_{[t,+\infty)} g, \quad f=M_{\Theta} g \in \mathscr{K}_{\Theta}, \quad t \geqslant 0,
\end{aligned}
$$

где $P_{[0, t]}$ и $P_{[t,+\infty)}$ есть проекторы на подпространства функций с носителем, принадлежашим $[0, t]$ и $[t,+\infty)$ соответственно. Таким образом, мы приходим к модели марковского коцикла, приведенной в [4].

Теорема 2. Пусть действие $\widetilde{U}_{t}$ продолэено в $\left(\mathscr{K}_{\Theta} \oplus \mathscr{K}_{-}\right)^{\perp}$, так что получивииеся операторы образуют сильно непрерывную группу унитарных операторов в $\mathscr{K}$. Тогда семейство унитарных операторов $W_{t}=\widetilde{U}_{t} U_{-t}, t \geqslant 0$, определяет марковский $U$-коиикл.

Для доказательства теоремы достаточно заметить, что условие (M) вьполнено в силу того, что $\left.\widetilde{U}_{t}\right|_{\mathscr{K}_{-}}=U_{t}, t \geqslant 0$.

Пусть $U$ есть сильно непрерывная группа унитарных операторов в $\mathscr{K}$ с генератором $\mathscr{L}_{0}=a \partial / \partial x$ и $\widetilde{U}-$ групша унитарных операторов, определяемая обобщенным решением задачи $(1),(2)$ с генератором $\mathscr{L}$ (см. замечание к теореме 1$)$. Группа $U$ унитарна эквивалентна группе сдвигов на прямой с точностью до изменения масштаба. Из формул (13) и (15) следует, что оператор, осуществляющий соответствующее унитарное отображение, можно подобрать таким образом, чтобы подпространство $L_{2}\left(\Omega_{2}\right)$ перешло в $\mathscr{K}_{-}$в случае $a>0$ и $L_{2}\left(\Omega_{1}\right)$ перешло в $\mathscr{K}_{-}$в случае $a<0$. Поскольку $\widetilde{U}_{t} f=U_{t} f, f \in L_{2}\left(\Omega_{2}\right)$, $t \geqslant 0$, при $a>0$, и $\widetilde{U}_{t} f=U_{t} f, f \in L_{2}\left(\Omega_{1}\right), t \geqslant 0$, при $a<0$, получаем согласно (13) и $(15)$, что коцикл $W_{t}=\widetilde{U}_{t} U_{-t}, t \in \mathbb{R}$, удовлетворяет условию (M) и является марковским. Таким образом, мы доказали следующее утверждение.

Теорема 3. Обобщенное решение задачи (1), (2) определяет такую группу унитарных операторов $\widetilde{U}$, что семейство унитарных операторов $W_{t}=\widetilde{U}_{t} U_{-t}, t \in \mathbb{R}$, где $U_{t}=e^{t a \frac{\partial}{\partial x}}$, образует марковский коиикл. 


\section{СПИСОК ЦИТИРОВАННОЙ ЛИТЕРАТУРЫ}

[1] Уэрт Ч., Томсон Р. Физика твердого тела. М.: Мир, 1966.

[2] Гитман Д. М., Тютин И. Д. Каноническое квантование полей со связями. М.: Наука, 1986.

[3] Amosov G. G. On Markovian cocycle perturbations in classical and quantum probability // Int. J. Math. Math. Sci. 2003. V. 54. P. 3443-3468.

[4] Lindsay J. M., Wills S. J. Fock space Markovian cocycles: their representation, generation and dilation // Canad. Math. Soc. Conf. Proc. 2000. V. 29. P. 455-470.

[5] Liebscher V. How to generate Markovian cocycles on boson Fock space // Infin. Dimen. Anal. Quantum Probab. Rel. Top. 2001. V. 4. P. 215-219.

[6] Фикера $Г$. К единой теории краевых задач для эллиптико-параболических уравнений второго порядка // Математика. 1963. Т. 164. С. 99-121.

[7] Сакбаев В. ЖК. О постановке задачи Коши для уравнения Шрёдингера, вырождающегося на полупространстве // ЖВМиМФ. 2002. Т. 42. №11. С. 1718-1729.

[8] Сакбаев В. ЖК. О свойствах решений задачи Коши для уравнения Шрёдингера, вырождающегося на полупрямой // Современная математика и ее приложения. Труды конференции "Суздаль-4". Т. 10. Суздаль, 2003. С. 176-192.

[9] Михайлов В. П. Дифференциальные уравнения в частных производных. М.: Наука, 1983.

[10] Владимиров В. С. Уравнения математической физики. М.: Наука, 1971.

[11] Рид М., Саймон Б. Современные методы математической физики. Т. 1. М.: Мир, 1977.

[12] Parthasarathy K. R. An Introduction to Quantum Stochastic Calculus. Basel: Birkhauser, 1992.

[13] Чеботарев А. М. Что такое квантовое стохастическое уравнение с точки зрения функционального анализа? // Матем. заметки. 2002. Т. 71. № 3. С. 448-469.

[14] Bhat B. V. R. Cocycles of CCR flows // Mem. Amer. Math. Soc. 2001. № 709. P. 1-114.

[15] Bhat B. V. R. Minimal isometric dilations of operator cocycles // Integr. Equat. Operat. Theory. 2002. V. 42. P. 125-141.

[16] Amosov G. G. Cocycle perturbation of quasifree algebraic $K$-flow leads to required asymptotic dynamics of associated completely positive semigroup // Infin. Dimen. Anal. Quant. Probab. Rel. Top. 2000. V. 3. P. 237-246.

[17] Никольский Н. К. Лекции об операторе сдвига. М.: Наука, 1980.

[18] Адамян В. М., Аров Д. З. Об одном классе операторов рассеяния и характеристических оператор-функций сжатий // Докл. АН СССР. 1965. Т. 160. С. 9-12.

[19] Адамян В. М., Аров Д. З. Об операторах рассеяния и полугруппах сжатий в гильбертовом пространстве // Докл. АН СССР. 1965. Т. 165. С. 9-12. 